\title{
Dietary Supplement with Vitamin C Prevents Nitrate Tolerance
}

\author{
Eberhard Bassenge, Nelli Fink, Mikhail Skatchkov, and Bruno Fink \\ Institute of Applied Physiology, University of Freiburg, Hermann-Herder-Str 7, D-79104 Freiburg, Germany
}

\begin{abstract}
Enhanced formation of superoxide radicals has been proposed to play a major role in the development of nitrate tolerance in humans. We tested the effects of vitamin C (Vit-C) supplementation on glyceroltrinitrate (GTN)-induced hemodynamic effects during 3-d nonintermittent transdermal administration of GTN $(0.4 \mathrm{mg} / \mathrm{h})$ in nine healthy subjects. Tolerance development was monitored by changes in arterial pressure, dicrotic digital pulse pressure, and heart rate. Studies with GTN, Vit-C, or GTN/Vit-C were successively carried out at random in three different series in the same subjects. GTN treatment caused an immediate rise in arterial conductivity (a/b ratio of dicrotic pulse), but within $2 \mathrm{~d}$ of initiating GTN, the $a / b$ ratio progressively decreased and reached basal levels. In addition, there was a progressive loss of the orthostatic decrease in blood pressure. However, coadministration of Vit-C and GTN fully maintained the GTN-induced changes in the orthostatic blood pressure, and the rise of $\mathrm{a} / \mathrm{b}$ ratio was augmented by $310 \%$ for the duration of the test period. Changes in vascular tolerance in GTN-treated subjects were paralleled by upregulation of the activity of isolated platelets, which was also reversed by Vit-C administration. These findings demonstrate that dietary supplementation with Vit-C eliminates vascular tolerance and concomitant upregulation of ex vivo-washed platelet activity during long-term nonintermittent administration of GTN in humans. (J. Clin. Invest. 1998. 102:6771.) Key words: arterial conductivity - soluble guanylyl cyclase activity - platelet aggregation - membrane microviscosity $\bullet$ cyclic guanylyl monophosphate
\end{abstract}

\section{Introduction}

Organic nitrates are used to treat a large variety of cardiovascular diseases, including myocardial ischemia (1); however, most nitrovasodilators share the disadvantage of inducing nitrate tolerance, especially during nonintermittent administration (2). Nitrate tolerance is a multifactorial phenomenon that includes both neurohormonal counterregulation and enhanced responses to vasoconstrictor agonists (3). Despite many hypotheses regarding induction of nitrate tolerance, the underly-

Address correspondence to Professor Dr. Eberhard Bassenge, Institute of Applied Physiology, University of Freiburg, HermannHerder-Str 7, D-79104 Freiburg, Germany. Phone: +49-761-2035424; FAX: +49-761-2035428; E-mail: angphys@ruf.uni-freiburg.de

Received for publication 19 June 1997 and accepted in revised form 27 April 1998.

J. Clin. Invest.

(C) The American Society for Clinical Investigation, Inc.

0021-9738/98/07/0067/05 \$2.00

Volume 102, Number 1, July 1998, 67-71

http://www.jci.org ing mechanisms have not been clearly defined. Recently Münzel and coworkers observed that the metabolism of glyceroltrinitrate $(\mathrm{GTN})^{1}$ was associated with enhanced superoxide production both in vitro and in vivo during tolerance, probably as a result of enhanced activity of NADPH oxidoreductases (4-6). Furthermore, they postulated that the increased flux of superoxide acted as the final mediator of nitrate tolerance by inactivating NO released either from the administered drug or the endothelium $(6,7)$.

In previous studies we attempted to suppress the superoxide-mediated inactivation of NO that is associated with GTN tolerance by examining the efficacy of various compounds that might intercept the free radical (8-11). Of the various antioxidants tested, including ascorbate (Vit-C), $\alpha$-tocopherol (VitE), $N$-acetyl-cysteine, and dimethyl sulfoxide, Vit-C proved to be most efficient in our preliminary studies (8-12). Similarly, other reports have demonstrated that endothelial vasomotor dysfunction associated with the oxidative stresses encountered in a variety of disease states (including non-insulin-dependent diabetes, coronary artery disease, hypertension, hypercholesterolemia, and smoking) can be corrected by vitamin $\mathrm{C}$ administration (13-17). Given these findings, the aim of this study was to test the effects of dietary supplementation with Vit-C on the development of GTN tolerance in human subjects.

\section{Methods}

The study was performed with nine healthy volunteers (two females, seven males) with a mean age of $38 \mathrm{yr}$ (range 21-56) and a mean body weight of $74 \mathrm{~kg}$ (range 56-83), and was carried out according to the Helsinki Declaration recommendations for clinical trials in humans. All subjects were tested with an $0.8-\mathrm{mg}$ GTN capsule given sublingually, and all responded with a $>40 \%$ increase in the $\mathrm{a} / \mathrm{b}$ ratio of the dicrotic digital pulse (see below for details).

Study protocol. In a blinded fashion, the nine subjects received (a) two successive GTN transdermal delivery systems (TDS) daily $(0.4 \mathrm{mg} / \mathrm{h}$ of GTN for $12 \mathrm{~h}$ ) and an oral placebo (instead of Vit-C) three times daily for $3 \mathrm{~d}$; (b) GTN-TDS twice daily with 2 Vit-C capsules $(500 \mathrm{mg}$, retard delivery; Cetebe Smith Kline, Beecham, Herrenberg, Germany) three times daily for $3 \mathrm{~d}$; or (c) placebo-TDS twice daily and two capsules of Vit-C three times daily for $3 \mathrm{~d}$. Cardiovascular parameters and digital pulse were registered throughout the study (photoelectric sensor; Hellige, Freiburg, Germany). Before the subjects were admitted to the study, their hemodynamic responses to a 0.4-mg GTN capsule per os were checked. The minimal drop in systolic blood pressure required for the subject's acceptance was $8 \mathrm{mmHg}$. Blood pressure was continuously recorded using a Dinamap XL (Johnson \& Johnson Medical GmbH, Norderstedt, Germany) both in the recumbent position and after an orthostatic challenge following a 10-min resting state (changes in the dicrotic pulse pressure waves a/b ratio sensitively reflect changes in arterial conductivity [18]). This approach has been successfully validated with regard to a parameter of left ventricular filling indicated by the changes in diastolic pulmonary artery pressure with a correlation coefficient of 0.96 (19).

1. Abbreviations used in this paper: GTN, glyceroltrinitrate; TDS, transdermal delivery system. 
Vit-C determinations. Vit-C concentrations in plasma were measured using HPLC and an electrochemical detector according to a modified method described by Pachla et al. (20).

Platelet isolation and aggregation studies. Blood was drawn from the cubital vein into citric acid solution $(6: 1 \mathrm{vol} / \mathrm{vol})$, and platelet suspensions were prepared according to Bassenge et al. (9). Platelets were counted microscopically, and adjusted to a final concentration of $120,000 \mathrm{cells} / \mu \mathrm{l}$. Thrombin-induced platelet aggregation was monitored in a two-channel aggregometer Elri-840 (ELRI, Milano, Italy) with the peak increase in light transmission obtained within 5 min after stimulation (21). All parameters were measured at an external $\mathrm{Ca}^{2+}$ concentration of $1 \mathrm{mM}$ after equilibration of platelets with a buffer for $20 \mathrm{~min}$.

Measurements of $\left[\mathrm{Ca}^{2+}\right]_{i}$. Changes in $\left[\mathrm{Ca}^{2+}\right]_{i}$ were determined spectrofluorometrically (SFM-25; Kontron Elektronik, Neufahrn, Germany) using Fura 2 AM dye (22) as described by Bassenge et al. (9) in $10 \mathrm{mM}$ Hepes buffer containing $1 \mathrm{mM}$ calcium.

Microviscosity measurements. Membrane fluidity of platelet plasma membranes was determined as described (23).

Cyclic GMP assay. The cGMP levels of washed platelets was determined in supernatants after neutralization with bicarbonate (24) using a radioimmunassay (cGMP [ $\left.{ }^{125} \mathrm{I}\right]$ RIA Kit; BioTrend, Cologne, Germany; reference 23). To determine cGMP content in phosphodiesterase-inhibited platelets, cells were incubated with $20 \mu \mathrm{M}$ zaprinast for $10 \mathrm{~min}(25)$.

Calculations. All values are expressed as mean \pm SEM. Statistical significance was determined by Student's $t$ test for paired data. Two groups of data were considered to be significantly different at a $P$ value $<0.05$.

\section{Results}

As demonstrated in Fig. 1 (top), daily application of GTN with two consecutive TDSs resulted in an early increase in arterial conductivity as assessed by vascular relaxation. $24 \mathrm{~h}$ after the start of GTN administration, the $\mathrm{a} / \mathrm{b}$ ratio of the digital pulse wave increased from $2.33 \pm 0.06$ to $2.52 \pm 0.06$ for an overall change of $8.2 \pm 2.5 \%(P<0.05)$. This dilation-induced response in conductivity progressively disappeared at the end of days 2 and 3 of GTN administration, thus indicating the onset of tolerance. In marked contrast, when Vit-C was administered with GTN (elevating plasma Vit-C levels fourfold from 2.90 .9 to $12.62 .5 \mu \mathrm{g} / \mathrm{ml}$ ), this decline in the GTN-induced elevation of arterial conductivity was prevented (Fig. 1). Indeed, the vasodilator/conductivity responses evoked by GTN in the presence of Vit-C supplementation were potentiated throughout the 3-d observation period (e.g., $24.5 \pm 1.7 \%$ vs. control at day 1 , compared with $8.2 \pm 1.5 \%$ without Vit-C; $P<0.05 ; n=$ 9), but returned to control levels when GTN administration was discontinued (Fig. 1). Vit-C supplementation alone caused no significant increase in conductivity (a/b ratio) at day 1 $(4.1 \pm 0.3 \%, P>0.05)$ or over the remaining period of Vit-C administration (Fig. 1).

In addition to the changes noted in arterial conductivity, GTN-evoked dilator responses were also reflected by a significant drop in systolic blood pressure after orthostatic challenge, which disappeared progressively during tolerance development (Fig. 1, middle). In marked contrast, the decrease in systolic pressure after orthostatic challenge was maintained during coadministration of GTN and the Vit-C supplements (Fig. 1). Parallel to the orthostatic systolic pressure drop, an increase in heart rate was observed in the GTN-Vit-C group, which was maintained over the course of the 3-d observation period (Fig. 1, bottom). This result is in contrast to GTN treat-
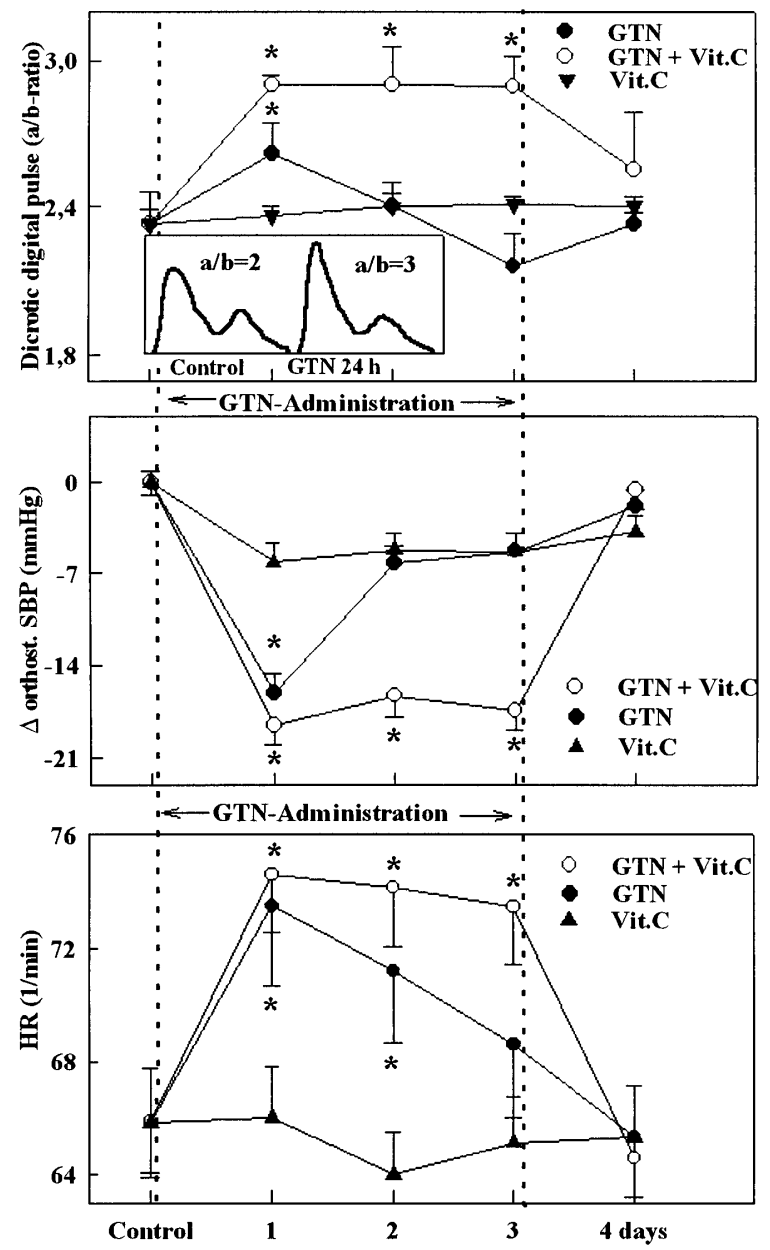

Figure 1. Effect of 3-d nonintermittent administration of GTN on vascular conductivity measured as relaxation-evoked changes in the $\mathrm{a} / \mathrm{b}$ ratio (inset) of the dicrotic finger pulse (top), in simultaneously elicited changes in systolic blood pressure induced by orthostatic challenge (orthostatic systolic blood pressure, middle) and heart rate $(H R$, bottom $)$. Data are mean \pm SEM. $* P<0.05$ vs. control.

ment without Vit-C supplementation, which elicited a progressive reduction in heart rate that led to tolerance after $3 \mathrm{~d}$ (Fig. 1, bottom). Significantly, Vit-C alone did not cause such changes in blood pressure or heart rate (Fig. 1).

To determine whether changes in vascular function were associated with alterations in platelet reactivity ex vivo, washed suspensions of platelets were recovered from each of the groups over the 4-d test period. As shown in Fig. 2, thrombin-induced increases in intracellular calcium $\left(\Delta\left[\mathrm{Ca}^{2+}\right]_{i}\right)$ rose progressively during GTN administration from $494 \pm 60 \mathrm{nM}$ to a peak of $741 \pm 37 \mathrm{nM}$ at day 3 . However, when Vit-C was coadministered with GTN, the increase in $\Delta\left[\mathrm{Ca}^{2+}\right]_{\mathrm{i}}$ corresponded to that observed in control platelets (Fig. 2). Vit-C alone caused a nonsignificant decrease in $\Delta\left[\mathrm{Ca}^{2+}\right]_{i}$ from $481 \pm 20$ to $425 \pm 18 \mathrm{nM}(P>0.05, n=9)$ at day 1 . Increases in $\Delta\left[\mathrm{Ca}^{2+}\right]_{\mathrm{i}}$ were also associated with parallel increases in platelet aggregation from $29.2 \pm 2.8 \%$ before GTN treatment to $85.4 \pm$ $8.5 \%$ at day 3 of GTN treatment (Fig. 2, bottom). Similarly, platelet membrane microviscosity increased from $1.65 \pm 0.05$ poise for controls to $1.92 \pm 0.09$ poise in the GTN-treated 

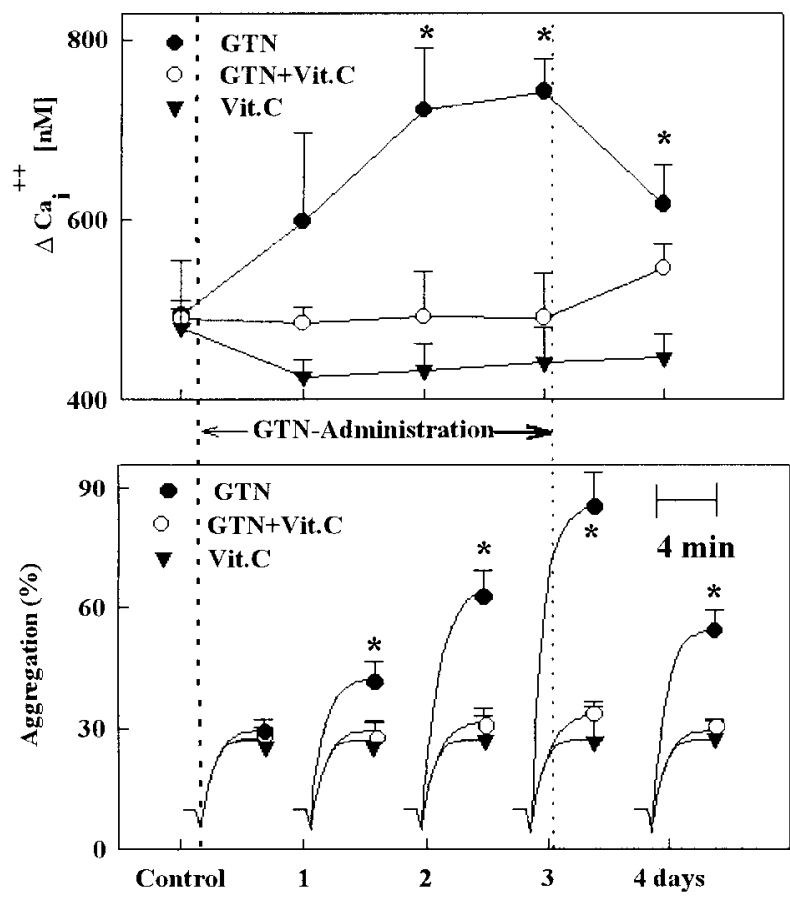

Figure 2. Effect of 3-d nonintermittent GTN administration on thrombin-induced changes in $\mathrm{Ca}^{2+}{ }_{\mathrm{i}}$ (top) and in thrombin-induced aggregation responses demonstrated by superimposed aggregation curves (light transmission) showing the median \pm SEM (filled symbols, bottom). Data are obtained with washed nonstimulated ex vivo platelets. Data are mean \pm SEM, $* P<0.05$ vs. control.

group $(n=9, P<0.01)$. In each case, dietary supplementation with Vit-C prevented the GTN-induced effects on platelet function. That is, increases in platelet responses to thrombin decreased to those seen in controls (Fig. 2, bottom) while platelet membrane viscosity returned to control values (i.e., $1.64 \pm 0.06$ at control, and $1.70 \pm 0.07$ poise at day 3 ; no significant difference; $P>0.05, n=6$ ). Vit-C alone (i.e., without GTN) caused an insignificant reduction in thrombin-stimulated aggregation from $19.2 \pm 2.8 \%$ at control to $18.7 \pm 2.1 \%$ at day 1 (Fig. 2), and did not affect membrane viscosity (1.65 \pm 0.05 at control vs. 1.680 .06 during Vit-C; $P>0.4$ ).

The increases observed in aggregation with GTN treatment also coincided with a progressive decrease in platelet basal cGMP levels by $33.8 \pm 7.1 \%$ at day 3 (Fig. 3, top). While cGMP levels were increased transiently during GTN/Vit-C treatment, values returned to control levels by day 3 . Vit-C did not affect basal cGMP-release. In the presence of the phosphodiesterase (PDE) inhibitor zaprinast, these trends in cGMP levels were not significantly affected (Fig. 3, bottom). Vit-C in the presence of phosphodiesterase inhibition did not affect basal cGMP-release.

\section{Discussion}

Documentation of tolerance. In this study, we have demonstrated that the vasodilatory changes associated with nitrate tolerance can be circumvented by dietary supplementation with Vit-C. Tolerance was documented by (a) monitoring changes in the $\mathrm{a} / \mathrm{b}$ ratio (a sensitive and reproducible parameter corresponding to arterial conductivity [19]); and (b) by monitoring orthostatic changes in systolic blood pressure and heart rate. At the end of $3 \mathrm{~d}$ of continuous GTN administration, early changes in each of these parameters had returned to control levels, documenting the development of tolerance. However, during coadministration of Vit-C, the original GTNinduced hemodynamic changes were maintained.

The mechanism(s) underlying nitrate tolerance remain undefined, and have included decreased biotransformation of GTN to its active metabolite(s), alterations in neurohumoral adaptation, and changes in guanylyl cyclase activity/activation $(4,7,13-17)$. However, recent attention has focused the ability of GTN to augment directly or indirectly the activity of the vessel wall-associated NADPH oxidoreductases that generate the superoxide anion $(4,7)$. In turn, superoxide has been postulated to inactivate the vasorelaxant properties of nitric oxide, as well as to promote formation of potentially deleterious metabolites, e.g., peroxynitrite $(4,7,13-17)$. Indeed, recent studies indicate that GTN metabolism in the tolerant state is accompanied by an increase in urinary excretion of nitrotyrosine (26). While the means by which ascorbate prevents tolerance remain to be determined, others have suggested that Vit-C could $(a)$ directly affect superoxide production by the NADPH oxidoreductase; $(b)$ scavenge superoxide directly, and thereby decrease nitric oxide inactivation; $(c)$ increase ni-

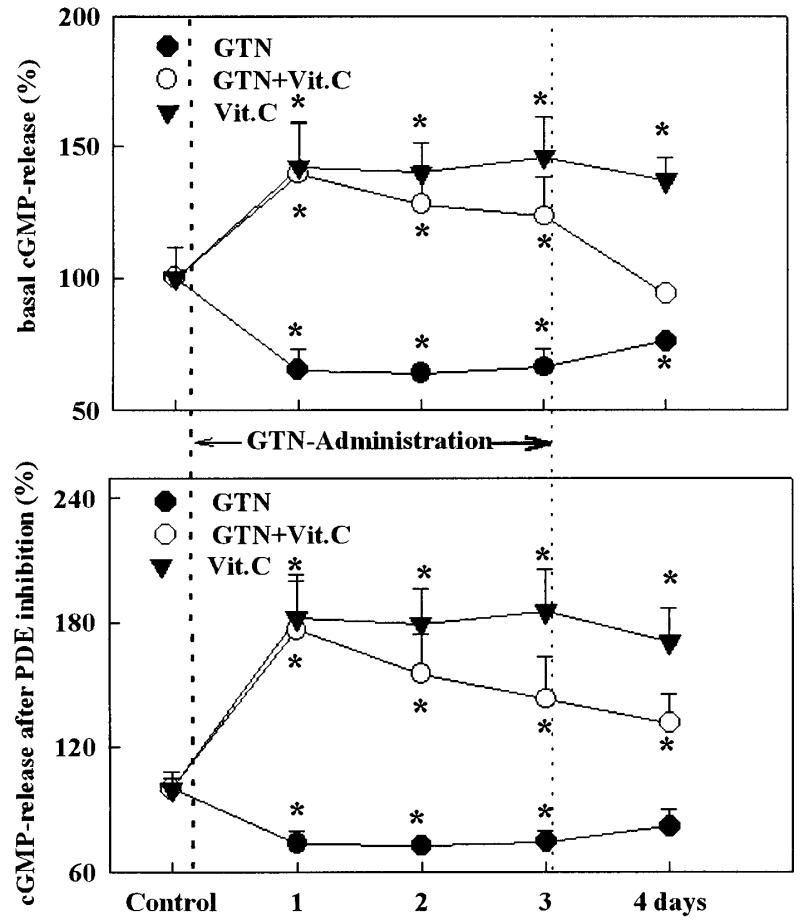

Figure 3. Effect of 3-d nonintermittent GTN administration on unstimulated basal cGMP-release in the presence or absence of phosphodiesterase inhibition by zaprinast. (Top) Unstimulated basal release of cGMP during GTN administration, during GTN with additional Vit-C, and during Vit-C administration. (Bottom) cGMP levels under identical drug applications in the presence of phosphodiesterase inhibition by zaprinast. Data are mean \pm SEM. $* P<0.05$ vs. control. 
trosothiol formation by protecting plasma thiols from oxidation; and $(d)$ suppress peroxynitrite-mediated inactivation of target enzymes (4, 7, 13-17, 27-29). Other factors, including possible effects on GTN biotransformation, the neurohumoral axis, prostacyclin generation, leukocyte aggregation, oxidized LDL generation, or platelet-activating factor mimetics, cannot be ruled out (30-36).

Assessment of ex vivo platelet activity as a sensitive marker for nitrate tolerance. An interesting observation in this study was the existence of an unexpected upregulation in the activity of washed platelets recovered from individuals undergoing GTN treatment. In animal studies, in vivo administration of organic nitrates exerts antiplatelet effects that can be monitored in vitro (37). Interestingly, however, hemodynamic tolerance was not always associated with a loss of antiaggregatory effects on platelets (37). While the reasons underlying these differences have not been defined, our studies demonstrate that GTN tolerance in humans is associated with profound changes in platelet function that can be reversed by Vit-C supplementation. The in vivo significance of ex vivo decreases in cGMP and associated increases in thrombin-induced $\mathrm{Ca}^{2+}$ or platelet aggregation remain to be determined, but the data suggest that these parameters could be used to monitor tolerance induction. In any case, it is interesting that the washed platelets that are no longer exposed to GTN, nitric oxide, or Vit-C appear to be imprinted from their in vivo exposure to the organic nitrate and its downstream effectors.

In conclusion, we have shown that tolerance to nonintermittent GTN administration in humans can be circumvented by dietary supplementation with vitamin C. Given the ability of Vit-C to reverse endothelial vasomotor dysfunction in a variety of vascular disease states that have been associated with increases in vessel wall-derived superoxide production $(4,7$, 13-17), we hypothesize that these nitric oxide-protective effects are a consequence of Vit-C's antioxidant properties. These observations are also in keeping with preliminary reports that have demonstrated similar effects in GTN-tolerant patients receiving other antioxidants, such as vitamin E (3840). Taken together, these studies suggest that administration of vitamin $\mathrm{C}$ may prove useful for overcoming the tolerant state induced during prolonged administration of nitrovasodilators in acute ischemic syndromes or congestive heart failure. In addition, as progressive upregulation in the activity of washed ex vivo platelets coincides with vascular tolerance, these simple assays may serve as useful clinical markers to assess further the underlying mechanisms associated with the induction or reversal of nitrate tolerance.

\section{Acknowledgments}

This study was supported by the German Heart Foundation (Deutsche Herzstiftung). We are grateful to H. Siegel, O. Sommer and R. Burzan for their excellent technical assistance, and to L. Böhm for her secretarial support.

\section{References}

1. Abrams, J. 1992. Use of nitrates in ischemic heart disease. Curr. Probl. Cardiol. 17:481-542.

2. Rudolph, W., J. Dirschinger, F. Kraus, G. Reiniger, and D. Hall. 1990. Nitrate therapy in patients with coronary artery disease, preparations and doses with and without development of tolerance. Z. Kardiol. 79(Suppl. 3):57-65.

3. Münzel, T., A. Giaid, S. Kurz, D.J. Stewart, and D.G. Harrison. 1995. Ev- idence for a role of endothelin 1 and protein kinase $\mathrm{C}$ in nitroglycerin tolerance. Proc. Natl. Acad. Sci. USA. 92:5244-5248.

4. Münzel, T., H. Sayegh, B.A. Freeman, M.M. Tarpey, and D.G. Harrison. 1995. Evidence for enhanced vascular superoxide anion production in nitrate tolerance. J. Clin. Invest. 95:187-194.

5. Münzel, T., and E. Bassenge. 1996. Long-term angiotensin-converting enzyme inhibition with high-dose enalapril retards nitrate tolerance in large epicardial arteries and prevents rebound coronary vasoconstriction in vivo. Circulation. 93:2052-2058.

6. Rajagopalan, S., S. Kurz, T. Munzel, M. Tarpey, B.A. Freeman, K.K. Griendling, and D.G. Harrison. 1996. Angiotensin II-mediated hypertension in the rat increases vascular superoxide production via membrane NADH/ NADPH oxidase activation. Contribution to alterations of vasomotor tone. $J$. Clin. Invest. 97:1916-1923.

7. Münzel, T., S. Kurz, S. Rajagopalan, M. Thoenes, W.R. Berrington, J.A Thompson, B.A. Freeman, and D.G. Harrison. 1996. Hydralazine prevents nitroglycerin tolerance by inhibiting activation of a membrane-bound NADH oxidase. A new action for an old drug. J. Clin. Invest. 98:1465-1470.

8. Fink, B., and E. Bassenge. 1995. Exogenous NO in contrast to endogenous NO is subject to tolerance due to enhanced $\mathrm{O}_{2}$-radical formation resulting from impaired NO-scavenging. Pfluegers Arch. 429:R108.

9. Bassenge, E., and B. Fink. 1996. Tolerance to nitrates and simultaneous upregulation of platelet activity prevented by enhancing antioxidant state. Naunyn-Schmiedeberg's Arch. Pharmacol. 353:363-367.

10. Bassenge, E., and B. Fink. 1996. Suppression of nitrate induced tolerance by vitamin $\mathrm{C}$ and other antioxidants. In The Biology of Nitric Oxide, Part 5. S. Moncada, J. Stamler, S. Gross, and E.A. Higgs, editors. Portland Press Ltd., London. 198.

11. Münzel, T., J. Holtz, A. Mülsch, D.J. Stewart, and E. Bassenge. 1989. Nitrate tolerance in epicardial arteries or in the venous system is not reversed by $\mathrm{N}$-acetylcysteine in vivo, but tolerance-independent interactions exist. $\mathrm{Circu}$ lation. 79:188-197.

12. Skatchkov, M., B. Fink, S. Dikalov, O. Sommer, and E. Bassenge. 1996. Antioxidant mediated prevention of nitrate tolerance is achieved through immediate inactivation of peroxinitrite. In The Biology of Nitric Oxide: Part 5. S. Moncada, J. Stamler, S. Gross, and E.A. Higgs, editors. Portland Press Ltd. London. 199.

13. Ting, H.H., F.K. Timimi, K.S. Boles, S.J. Creager, P. Ganz, and M.A. Creager. 1996. Vitamin C improves endothelium-dependent vasodilation in patients with non-insulin-dependent diabetes mellitus. J. Clin. Invest. 97:22-28.

14. Levine, G.N., B. Frei, S.N. Koulouris, M.D. Gerhard, J.F.J. Keaney, and J.A. Vita. 1996. Ascorbic acid reverses endothelial vasomotor dysfunction in patients with coronary artery disease. Circulation. 93:1107-1113.

15. Solzbach, U., B. Hornig, M. Jeserich, and H. Just. 1997. Vitamin C improves endothelial dysfunction of epicardial coronary arteries in hypertensive patients. Circulation. 96:1513-1519.

16. Ting, H.H., F.K. Timimi, E.A. Haley, M.A. Roddy, P. Ganz, and M.A. Creager. 1997. Vitamin C improves endothelium-dependent vasodilation in forearm resistance vessels of humans with hypercholesterolemia. Circulation. 95:2617-2622.

17. Heitzer, T., H.J. Just, and T. Münzel. 1996. Antioxidant vitamin C improves endothelial dysfunction in chronic smokers. Circulation. 94:6-9.

18. Imhof, P.R., B. Ott, P. Frankhauser, L.C. Chu, and J. Hodler. 1980. Difference in nitroglycerin dose-response in the venous and arterial beds. Eur. J. Clin. Pharmacol. 18:455-460.

19. Buschmann, M., A. Wiegand, K. Schnellbacher, R. Bonn, A. Rehe, D. Trenk, E. Jähnchen, and H. Roskamm. 1993. Comparison of the effects of two different galenical preparations of glyceryl trinitrate on pulmonary artery pressure and on the finger pulse curve. Eur. J. Clin. Pharmacol. 44:451-456.

20. Pachla, L.A., and P.T. Kissinger. 1979. Analysis of ascorbic acid by liquid chromatography with amperometric detection. Methods Enzymol. 62:15-24.

21. Ivanova, K., M. Schaefer, C. Drummer, and R. Gerzer. 1993. Effects of nitric oxide-containing compounds on increases in cytosolic ionized $\mathrm{Ca}^{2+}$ and on aggregation of human platelets. Eur. J. Pharmacol. Mol. Pharmacol. Sect. 244:37-47.

22. Grynkiewicz, G., M. Poenie, and R.Y. Tsien. 1985. A new generation of $\mathrm{Ca} 2+$ indicators with greatly improved fluorescence properties. J. Biol. Chem. 260:3440-3450.

23. Feijge, M.A.H., J.W.M. Heemskerk, and G. Hornstra. 1990. Membrane fluidity of non-activated and activated human blood platelets. Biochim. Biophys. Acta. 1025:173-178.

24. Fink, B., and E. Bassenge. 1997. Unexpected, tolerance-devoid vasomotor and platelet actions of pentaerytrityl tetranitrate. J. Cardiovasc. Pharmacol. 30:831-836.

25. Weber, A.A., H. Strobach, and K. Schrör. 1993. Direct inhibition of platelet function by organic nitrates via nitric oxide formation. Eur. J. Pharmacol. 247:29-37.

26. Skatchkov, M., L. Larina, A. Larin, N. Fink, and E. Bassenge. 1997. Urinary nitrotyrosine content as a marker of peroxynitrite-induced tolerance to organic nitrates. J. Cardiovasc. Pharmacol. Ther. 2:85-96.

27. Nunes, G.L., K. Robinson, A. Kalynych, S.B. King, D.S. Sgoutas, and B.C. Berk. 1997. Vitamins C and E inhibit O2- production in the pig coronary 
artery. Circulation. 96:3593-3601.

28. Beckman, J.S., H. Ischiropoulos, L. Zhu, M. van der Woerd, C. Smith, J. Chen, J. Harrison, J.C. Martin, and M. Tsai. 1992. Kinetics of superoxide dismutase- and iron-catalyzed nitration of phenolics by peroxynitrite. Arch. Biochem. Biophys. 298:438-445.

29. Braughler, J.M. 1983. Soluble guanylate cyclase activation by nitric oxide and its reversal. Involvement of sulfhydryl group oxidation and reduction. Biochem. Pharmacol. 32:811-818.

30. Dikalov, S., B. Fink, O. Sommer, and E. Bassenge. 1998. Formation of reactive oxygen species in various vascular cells during nitroglycerin metabolism. J. Cardiovasc. Pharmacol. Ther. 3:1-11.

31. Toivanen, J.L. 1987. Effects of selenium, vitamin E and vitamin C on human prostacyclin and thromboxane synthesis in vitro. Prostaglandins Leukot. Med. 26:265-280.

32. Beetens, J.R., M.C. Coene, A. Veheyen, L. Zonnekeyn, and A.G. Herman. 1986. Vitamin C increases the prostacyclin production and decreases the vascular lesions in experimental atherosclerosis in rabbits. Prostaglandins. 32: $335-352$.

33. Lehr, H.-A., B. Frei, and K.-E. Arfors. 1994. Vitamin C prevents cigarette smoke-induced leukocyte aggregation and adhesion to endothelium in vivo. Proc. Natl. Acad. Sci. USA. 91:7688-7692.

34. Retsky, K.L., M.W. Freeman, and B. Frei. 1993. Ascorbic acid oxidation product(s) protect human low density lipoprotein against atherogenic modification. Anti- rather than prooxidant activity of vitamin $\mathrm{C}$ in the presence of transition metal ions. J. Biol. Chem. 268:1304-1309.

35. Lehr, H.A., A.S. Weyrich, R.K. Saetzler, A. Jurek, K.E. Arfors, G.A. Zimmerman, S.M. Prescott, and T.M. McIntyre. 1997. Vitamin C blocks inflammatory platelet-activating factor mimetics created by cigarette smoking. J. Clin. Invest. 99:2358-2364.

36. Diaz, M.N., B. Frei, J.A. Vita, and J.F. Keaney, Jr. 1997. Antioxidants and atherosclerotic heart disease. N. Engl. J. Med. 337:408-416.

37. Booth, B.P., S. Jacob, J.A. Bauer, and H.L. Fung. 1996. Sustained antiplatelet properties of nitroglycerin during hemodynamic tolerance in rats. $J$. Cardiovasc. Pharmacol. 28:432-438.

38. Watanabe, H., M. Kakihana, S. Ohtsuka, and Y. Sugishita. 1996. Randomized, double-blind, placebo-controlled study of supplemental vitamin E on attenuation of the development of nitrate tolerance. Circulation. 94:503-504.

39. Bassenge, E., N. Fink, and B. Fink. 1998. Effects of oxidant stress circumvented by antioxidants vitamin $\mathrm{C}$ or by $\beta$-blocker carvedilol metabolite BM 910228. Pfluegers Arch. Eur. J. Physiol. 435:R74.

40. Watanabe, H., M. Kakihana, S. Ohtsuka, and Y. Sugishita. 1997. The preventive effect of carvedilol on nitrate tolerance in patients with congestive heart failure. 19th Congr. Eur. Soc. Cardiol. Stockholm, Aug. 18:400. 